\title{
Spin diffusion and injection in semiconductor structures: Electric field effects
}

\author{
Z. G. Yu and M. E. Flatté \\ Department of Physics and Astronomy, University of Iowa, Iowa City, Iowa 52242
}

(Dated: October 29, 2018)

\begin{abstract}
In semiconductor spintronic devices, the semiconductor is usually lightly doped and nondegenerate, and moderate electric fields can dominate the carrier motion. We recently derived a driftdiffusion equation for spin polarization in the semiconductors by consistently taking into account electric-field effects and nondegenerate electron statistics and identified a high-field diffusive regime which has no analogue in metals. Here spin injection from a ferromagnet (FM) into a nonmagnetic semiconductor (NS) is extensively studied by applying this spin drift-diffusion equation to several typical injection structures such as FM/NS, FM/NS/FM, and FM/NS/NS structures. We find that in the high-field regime spin injection from a ferromagnet into a semiconductor is enhanced by several orders of magnitude. For injection structures with interfacial barriers, the electric field further enhances spin injection considerably. In FM/NS/FM structures high electric fields destroy the symmetry between the two magnets at low fields, where both magnets are equally important for spin injection, and spin injection becomes locally determined by the magnet from which carriers flow into the semiconductor. The field-induced spin injection enhancement should also be insensitive to the presence of a highly doped nonmagnetic semiconductor $\left(\mathrm{NS}^{+}\right)$at the FM interface, thus $\mathrm{FM} / \mathrm{NS}^{+} / \mathrm{NS}$ structures should also manifest efficient spin injection at high fields. Furthermore, high fields substantially reduce the magnetoresistance observable in a recent experiment on spin injection from magnetic semiconductors.
\end{abstract}

PACS numbers: 72.25.Dc, 72.20.Ht, 72.25.Hg, 72.25.Mk.

\section{INTRODUCTION}

Semiconductor devices based on the control and manipulation of electron spin (semiconductor spintronics) have recently attracted considerable attention since the discovery of long spin relaxation times and large spin transport distanfes in semiconductors and various device structures 1 . In order to design and fabricate highperformance spintronic devices, a comprehensive understanding of spin transport and injection properties of semiconductors and heterostructures is needed.

In theoretical studies of spin transport and injection in

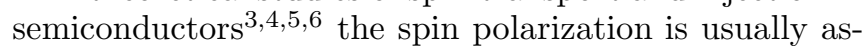
sumed to obey the same diffusion equation as in metals,

$$
\nabla^{2}\left(\mu_{\uparrow}-\mu_{\downarrow}\right)-\left(\mu_{\uparrow}-\mu_{\downarrow}\right) / L^{2}=0,
$$

where $\mu_{\uparrow(\downarrow)}$ is the electrochemical potential of up-spin (down-spin) electrons. In this diffusion equation, the electric field does not play any role, and spin polarization decays away on a length scale of $L$ from an injection point. This is reasonable for metals because the electric field $\mathbf{E}$ is essentially screened. For semiconductor spintronic devices, however, the semiconductor often is lightly doped and nondegenerate, and a moderate electric field can dominate the carrier motion. In fact, experiments have shown that electric fields-fian affect spin diffusion in semiconductors dramatically 6 a

In Ref. 10, we examined the role of electric field on spin transport in nondegenerate semiconductors and derived a drift-diffusion equation for spin polarization,

$$
\nabla^{2}\left(n_{\uparrow}-n_{\downarrow}\right)+\frac{e \mathbf{E}}{k_{B} T} \cdot \nabla\left(n_{\uparrow}-n_{\downarrow}\right)-\frac{n_{\uparrow}-n_{\downarrow}}{L^{2}}=0,
$$

where $n_{\uparrow(\downarrow)}$ is the deviation of up-spin (down-spin) electron density from its equilibrium value, $k_{B}$ the Boltzmann constant, and $T$ the temperature. This equation consistently takes into account electric-field effects and nondegenerate electron statistics. We identified a highfield diffusive regime which has no analogue in metals. This regime occurs for field as small as $1 \mathrm{~V} / \mathrm{cm}$ at low temperatures. Two distinct spin diffusion lengths now characterize spin motion, i.e., up-stream $\left(L_{u}\right)$ and downstream $\left(L_{d}\right)$ spin diffucion lengths. This is a further example of the analogy 11.12 between up/down-spin electron in semiconductor spin transport and majority/minority carriers in semiconductor charge transport, where the presence of electric field also results in two charge diffusion lengths 13 We applied this spin drift-diffusion equation to study spin injection from a ferromagnet into a semiconductor, and showed that the electric-field effects on spin injection could be described in terms of the two field-induced spin diffusion lengths in the semiconductor.

In this paper, first, we derive a more general driftdiffusion equation of spin polarization valid for both doped semiconductors and metals, and demonstrate the development from Eq. (1.2) to Eq. (1.1) as the system changes from nondegenerate to degenerate. We establish the relation between the electrochemical potential splitting and density imbalance of up-spin and down-spin electrons in nondegenerate systems, and clarify the two 
spin polarizations, i.e., spin polarization of current and spin polarization of density. We then use the spin driftdiffusion equation for nondegenerate systems, Eq. (1.2), to analyze several typical one-dimensional device geometries. We find that high fields also enhance spin injection from a ferromagnet to a semiconductor in structure with a spin-selective barrier. Rashba, 1 Smith and Silver, 5 Fert and Jaffrès, 6 and Flatté, Byers, and Lau in Ref. 2 have considered such a barrier in the low-field regime. The field enhancement and the interface enhancement of spin injection may reinforce each other to achieve high injection efficiencies in different structures.

Next, we study spin injection in sandwiched FM/NS/FM structures with and without spin-selective interfacial barriers. At low fields the two magnets are equally important to determine spin injection into the semiconductor and the spin injection efficiency is sensitive to the relative orientation of the two magnets. We find that in the high-field regime, this symmetry is broken and spin injection is locally determined by the magnet from which carriers are injected into the semiconductor. The spin injection efficiency can be enhanced by orders of magnitude by increasing the electric field for both parallel and anti-parallel orientation of the two magnets.

We further consider $\mathrm{FM} / \mathrm{NS}^{+} / \mathrm{NS}$ structures, where a highly doped nonmagnetic semiconductor $\left(\mathrm{NS}^{+}\right)$is placed near the magnet interface. Such a configuration is common in structures designed to overcome the Schottky barrier between a magnet and a semiconductor, and is intrinsic to FM/InAs, where densely occupied surface states form at the interface. We find that spin injection at the strong-field limit in such a structure is controlled by the total electric current flowing into semiconductors and insensitive to the distinction between semiconductors. Thus high fields can effectively enhance spin injection in such structures as well.

Finally we explore electric-field effects on magnetoresistance of a magnetic semiconductor (MS)/NS/MS structure. A magnetic semiconductor can have extremely large spin polarization at low temperatures and the magnetization can be easily adjusted by applying an external magnetic field. A large positive magnetoresistance have been observed in MS/NS/MS structures.14 We find that this magnetoresistance collapses in the high-field regime, suggesting a sensitive test of the electric-field effects on spin transport in semiconductors.

The paper is organized as follows. In Sec. II we review the general spin drift-diffusion equation in nondegenerate and degenerate systems and analyze the field-induced spin diffusion lengths. In Sec. III we investigate spin injection in FM/NS structures with an interfacial barrier. Sections IV and $\mathrm{V}$ contain results on spin injection in $\mathrm{FM} / \mathrm{NS} / \mathrm{FM}$ structures and in FM/NS/NS structures, respectively. Section VI is devoted to the electric-field effects on magnetoresistance in MS/NS/MS structures. In Sec. VII, we summarize our conclusions.

\section{ELECTRIC FIELD AND SPIN TRANSPORT}

In this section we derive a more general drift-diffusion equation for spin polarization valid in both degenerate and nondegenerate systems and discuss the electricfield effects on spin transport by analyzing the structure of this equation. Discussion of the drift-diffusion equations of carrier motion in semiconductors involving spin-dependent processes can be tracked back decades ago. Pierce et al. incorporated spin relaxation in a onedimension diffusion model of up-spinand down-spin carrier densities at zero electric field.15 Sogawa et al. investigated spin transport in wires with a set of complete drift-diffusion equations for minority carriers, which explicitly include spin-flipand recombination processes as well as the electric field.16 More recently Zutić et al. displayed such equations for both minority and majority carriers 17 Usually extensive numerical calculations are required to solve the set of drift-diffusion equations together with the Poisson's equation self-consistently. The spin drift-diffusion equation we derive is a single equation instead of a set of equations and can be solved analytically in several interesting geometries. The role of this spin drift-diffusion equation in spin transport is similar to that of the ambipolar drift-diffusion equation in charge transport.

\section{A. Drift-diffusion equation for spin polarization}

The system we consider here is $n$-doped ( $p$-doped systems can be analyzed similarly), which can be ferromagnetic or nonmagnetic. The analysis presented in this section is valid not only in doped semiconductors but also in metals. We assume that there is no space charge and the material is homogeneous. The current for up-spin and down-spin can be written as

$$
\begin{aligned}
& \mathbf{j}_{\uparrow}=\sigma_{\uparrow} \mathbf{E}+e D_{\uparrow} \nabla n_{\uparrow}, \\
& \mathbf{j}_{\downarrow}=\sigma_{\downarrow} \mathbf{E}+e D_{\downarrow} \nabla n_{\downarrow},
\end{aligned}
$$

which consists of the drift current and the diffusion one. Here $D_{\uparrow(\downarrow)}$ is the up-spin (down-spin) electron diffusion constant and $\sigma_{\uparrow(\downarrow)}$ the up-spin (down-spin) conductivity. The change of up-spin (down-spin) conductivity from its unperturbed value $\sigma_{\uparrow(\downarrow)}^{0}$ in the presence of spin polarization, $\Delta \sigma_{\uparrow(\downarrow)} \equiv \sigma_{\uparrow(\downarrow)}-\sigma_{\uparrow(\downarrow)}^{0}$, is assumed to be proportional to $n_{\uparrow(\downarrow)}$, the up-spin (down-spin) electron density deviation from its equilibrium value $n_{\uparrow(\downarrow)}^{0}$,

$$
\Delta \sigma_{\uparrow(\downarrow)}=n_{\uparrow(\downarrow)} e \nu_{\uparrow(\downarrow)} .
$$

Here the mobility $\nu_{\uparrow(\downarrow)}$ is independent of field and density over the range of density variation $n_{\uparrow(\downarrow)}$.

The continuity equations for up-spin and down-spin electrons in systems including spin-flip scattering process 
are

$$
\begin{aligned}
& \frac{\partial n_{\uparrow}}{\partial t}=-\frac{n_{\uparrow}}{\tau_{\uparrow \downarrow}}+\frac{n_{\downarrow}}{\tau_{\downarrow \uparrow}}+\frac{1}{e} \nabla \cdot \mathbf{j}_{\uparrow}, \\
& \frac{\partial n_{\downarrow}}{\partial t}=-\frac{n_{\downarrow}}{\tau_{\downarrow \uparrow}}+\frac{n_{\uparrow}}{\tau_{\uparrow \downarrow}}+\frac{1}{e} \nabla \cdot \mathbf{j}_{\downarrow},
\end{aligned}
$$

where $\tau_{\uparrow \downarrow}^{-1}\left(\tau_{\downarrow \uparrow}^{-1}\right)$ is the rate with which up-spin (downspin) electrons scatter to down-spin (up-spin) electrons. Here the recombination process is neglected because the system we consider is doped (unipolar). In steady state $\left[\partial n_{\uparrow(\downarrow)} / \partial t=0\right]$, we have

$$
\begin{aligned}
& \nabla \sigma_{\uparrow} \cdot \mathbf{E}+\sigma_{\uparrow} \nabla \cdot \mathbf{E}+e D_{\uparrow} \nabla^{2} n_{\uparrow}=\left(\frac{n_{\uparrow}}{\tau_{\uparrow \downarrow}}-\frac{n_{\downarrow}}{\tau_{\downarrow \uparrow}}\right) e, \\
& \nabla \sigma_{\downarrow} \cdot \mathbf{E}+\sigma_{\downarrow} \nabla \cdot \mathbf{E}+e D_{\downarrow} \nabla^{2} n_{\downarrow}=\left(\frac{n_{\downarrow}}{\tau_{\downarrow \uparrow}}-\frac{n_{\uparrow}}{\tau_{\uparrow \downarrow}}\right) e,
\end{aligned}
$$

where $\nabla \cdot \mathbf{E}=-e\left(n_{\uparrow}+n_{\downarrow}\right) / \epsilon$, and $\epsilon$ is the dielectric constant of the system.

For a homogeneous system without space-charge, $n_{\uparrow}+$ $n_{\downarrow}$ should be balanced by a local change of hole concentration. In doped systems, however, spin polarization can be reated without changing electrons or hole densities, $111 \mathrm{18}$ and therefore,

$$
n_{\uparrow}+n_{\downarrow}=0 .
$$

Care is required, however, to aypid setting $\nabla \cdot \mathbf{E}=0$ directly in Eqs. (2.5) and (2.6) 13 Instead we multiply Eq. (2.5) by $\sigma_{\downarrow}$ and Eq. (2.6) by $\sigma_{\uparrow}$, and substract one from the other, eliminating the terms containing $\nabla \cdot \mathbf{E}$. Only then do we set $n_{\uparrow}+n_{\downarrow}=0$. Now we have

$$
\nabla^{2}\left(n_{\uparrow}-n_{\downarrow}\right)+\frac{\nu}{e D} e \mathbf{E} \cdot \nabla\left(n_{\uparrow}-n_{\downarrow}\right)-\frac{n_{\uparrow}-n_{\downarrow}}{L^{2}}=0,
$$

where the effective mobility $\nu$ and the effective diffusion constant $D$ for spin polarization are

$$
\begin{aligned}
\nu & =\frac{\sigma_{\uparrow} \nu_{\downarrow}+\sigma_{\downarrow} \nu_{\uparrow}}{\sigma_{\uparrow}+\sigma_{\downarrow}}, \\
D & =\frac{\sigma_{\uparrow} D_{\downarrow}+\sigma_{\downarrow} D_{\uparrow}}{\sigma_{\uparrow}+\sigma_{\downarrow}} .
\end{aligned}
$$

And

$$
L=\sqrt{D \tau_{S}}
$$

is the intrinsic spin-diffusion length, where the spin relaxation time $\tau_{S}$ is defined via $\tau_{S}^{-1}=\tau_{\uparrow \downarrow}^{-1}+\tau_{\downarrow \uparrow}^{-1}$. Equations (2.9a) and 2.9b) indicate that the behavior of spin transport is controlled by the minority spin species. This is analogous to the ambipolar charge transport, where minority charge carriers determine the behavior of excess charge transport.13
For nonmagnetic systems, $\nu_{\uparrow}=\nu_{\downarrow}=\nu$ and $D_{\uparrow}=$ $D_{\downarrow}=D$. For ferromagnetic systems, $\nu$ and $D$ are approximately the mobility and the diffusion constant for the lower-conductivity spin species, usually the minority spins. We will assign the down-spin label to this species, so $\nu \simeq \nu_{\downarrow}$ and $D \simeq D_{\downarrow}$. Thus the coefficient of the second term in Eq. (2.8) can be approximated from the single-band form of the Einstein relation, 13

$$
\frac{\nu}{e D}=-\int_{0}^{\infty} N(\mathcal{E}) \frac{\partial f_{0}}{\partial \mathcal{E}} d \mathcal{E} / \int_{0}^{\infty} N(\mathcal{E}) f_{0}(\mathcal{E}) d \mathcal{E}
$$

where $\mathcal{E}$ is the energy measured from the bottom edge of the conduction band for the minority spin, $N(\mathcal{E})$ is the density of states for the minority spin and $f_{0}$ the distribution function. A more accurate evaluation of $\nu / e D$ when $\nu_{\uparrow} \neq \nu_{\downarrow}$ could not be done without knowledge of, e.g., $\nu_{\uparrow} / \nu_{\downarrow}$.

One special exception exists, however, for nondegenerate semiconductors, where $f_{0}$ has the Boltzmann form, $f_{0} \sim e^{-\mathcal{E} / k_{B} T}$, and $\nu / e D=1 / k_{B} T$. Thus we obtain Eq. (1.2) to describe the transport of $n_{\uparrow}-n_{\downarrow}$, the natural measure of the spin polarization in semiconductors. We emphasize that Eq. (1.2) is also valid for highly spinpolarized (including ferromagnetic) nondegenerate semiconductors.

For degenerate systems, $f_{0}$ in Eq. (2.11) should have the Fermi-Dirac form. In a three-dimensional (3D) system, $N(\mathcal{E})=A \mathcal{E}^{1 / 2}$, we have approximately

$$
\frac{\nu}{e D}=\frac{1}{2 k_{B} T} \frac{F_{-1 / 2}\left[\left(\varepsilon_{F}-\varepsilon_{\downarrow}^{b}\right) / k_{B} T\right]}{F_{1 / 2}\left[\left(\varepsilon_{F}-\varepsilon_{\downarrow}^{b}\right) / k_{B} T\right]},
$$

where $\varepsilon_{F}$ is the Fermi energy, $\varepsilon_{\downarrow}^{b}$ the bottom edge of the conduction band for the minority spin, and $F_{n}(\xi)=$ $\int_{0}^{\infty} d x x^{n}\left[e^{x-\xi}+1\right]^{-1} 13$ In a two-dimensional (2D) system, $N(\mathcal{E})$ is a constant,

$$
\frac{\nu}{e D}=\frac{1}{k_{B} T F_{0}\left[\left(\varepsilon_{F}-\varepsilon_{\downarrow}^{b}\right) / k_{B} T\right]\left[1+e^{-\left(\varepsilon_{F}-\varepsilon_{\downarrow}^{b}\right) / k_{B} T}\right]} .
$$

We can define a critical field

$$
E_{c} \equiv \frac{1}{e L}\left(\frac{\nu}{e D}\right)^{-1},
$$

such that when $E>E_{c}$, the drift term will be more important than the diffusive term in Eq. (2.8), and neglecting the electric-field effects on spin transport in this regime cannot be justified. In Fig. 1, we plot $E_{c}$ as a function of electron density for different temperatures in 2D and 3D $n$-doped GaAs using a typical spin diffusion length $L=2 \mu \mathrm{m}$ We can see that for electron densities ranging from $10^{15}$ to $10^{18} \mathrm{~cm}^{-3}$ the critical field $E_{c}$ is not beyond realistic fields under which spintronic devices operate. In particular, at low temperatures $E_{c}$ can be as low as $1 \mathrm{~V} / \mathrm{cm}$ in lightly and moderately doped semiconductors. Even for $100 \%$ spin polarized $n$-doped ZnMnSe, 


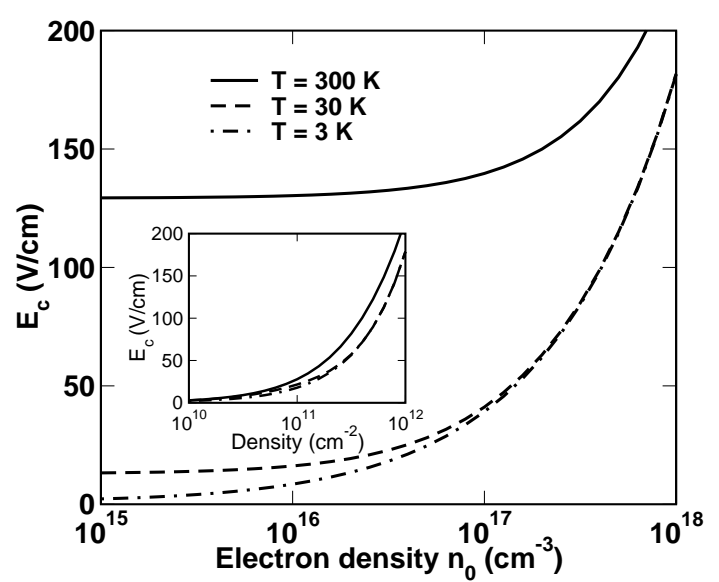

FIG. 1: Critical field $E_{c}$ as a function of electron density for different temperatures in $3 \mathrm{D}$ systems. The inset is for $2 \mathrm{D}$ systems. The effective electron mass $m^{*}=0.067 m_{0}$, where $m_{0}$ is the free electron mass.

$n_{0}=10^{18} \mathrm{~cm}^{-3}$, the drift term is relevant for $E>200$ $\mathrm{V} / \mathrm{cm}$ at $T<30 \mathrm{~K}$. Thus the electric field should be taken into account to properly interpret phenomena involving spin transport in both magnetic and nonmagnetic semiconductors.

For highly degenerate systems such as metals, in which $\varepsilon_{F}-\varepsilon_{\downarrow}^{b} \sim 10 \mathrm{eV} \gg k_{B} T$, from Eqs. (2.12) and (2.13), $\nu / e D=3 / 2\left(\varepsilon_{F}-\varepsilon_{\downarrow}^{b}\right)$ for a $3 \mathrm{D}$ system and $\nu / e D=$ $1 /\left(\varepsilon_{F}-\varepsilon_{\downarrow}^{b}\right)$ for a $2 \mathrm{D}$ system. For a typical spin diffusion length of metal, $L \sim 100 \mathrm{~nm}$, the field has to exceed $10^{6} \mathrm{~V} / \mathrm{cm}$ for the drift term to become comparable to the diffusion term in Eq. (2.8). Thus in metals under realistic fields the drift term can be neglected. Using the relation between the electrochemical potential, $\mu_{\uparrow(\downarrow)}$, and the nonequilibrium carrier density, $n_{\uparrow(\downarrow)}$, in a highly degenerate system

$$
n_{\uparrow(\downarrow)}=e N_{\uparrow(\downarrow)}\left(\varepsilon_{F}\right)\left[\mu_{\uparrow(\downarrow)}+e \psi\right],
$$

where $N_{\uparrow(\downarrow)}\left(\varepsilon_{F}\right)$ is the up-spin (down-spin) density of states at the Fermi energy, and $\mathbf{E}=-\nabla \psi$, we find that Eqs. (2.7) and (2.8) reduce to

$$
\nabla^{2}\left(\begin{array}{c}
\mu_{\uparrow} \\
\mu_{\downarrow}
\end{array}\right)=\frac{1}{L^{2}}\left(\begin{array}{cc}
\frac{\sigma_{\downarrow}}{\sigma_{\uparrow}+\sigma_{\downarrow}} & -\frac{\sigma_{\downarrow}}{\sigma_{\uparrow}+\sigma_{\downarrow}} \\
-\frac{\sigma_{\uparrow}}{\sigma_{\uparrow}+\sigma_{\downarrow}} & \frac{\sigma_{\uparrow}}{\sigma_{\uparrow}+\sigma_{\downarrow}}
\end{array}\right)\left(\begin{array}{c}
\mu_{\uparrow} \\
\mu_{\downarrow}
\end{array}\right),
$$

which is consistent with Eq. (2.18) in Ref. 19, the spin transport equation derived for metallic systems. It is straightforward to see that Eq. (11.1) is contained in Eq. 2.16). In deriving Eq. (2.16) we have assumed that the spin-dependent conductivity is proportional to the spin-dependent density of states at the Fermi level, $\sigma_{\uparrow} / \sigma_{\downarrow}=N_{\uparrow}\left(\varepsilon_{F}\right) / N_{\downarrow}\left(\varepsilon_{F}\right)$. The effects of electron-electron interaction (alterations of the spin stiffness, spin drag, etc.) will modify the value of $\nu / e D$, although for the temperatures of greatest interest and moderate density the corrections are small.20

Equation (1.2) provides a framework to understand spin transport in nondegenerate semiconductors, and Eqs. 2.82.13 in moderately degenerate semiconductors. We see that the electric field, unlike that in metals, plays a central role on spin transport. Comparing Eq. (1.2) with the drift-diffusion equation of minority carriers (electrons) in $p$-doped semiconductors, 13

$$
\nabla^{2}\left(n-n_{0}\right)+\frac{e \mathbf{E}}{k_{B} T} \cdot \nabla\left(n-n_{0}\right)-\frac{n-n_{0}}{L_{e}^{2}}=0,
$$

where $L_{e}$ is the intrinsic electron diffusion length, we find that Eq. (1.2) and Eq. (2.17) have the same structure and the electric field is expected to play a similar role in both situations. For minority carrier transport it is well-known that the electric field gives rise to two distinct charge diffusion lengths and considerably modifies minority charge injection.

\section{B. Spin diffusion lengths}

The spin drift-diffusion equation (2.8), together with the local charge neutrality constraint Eq. (2.7), dramatically alters the spin transport behavior in semiconductors from that expected from Eq. (1.1). The general solution to Eq. (1.1) (restricting variation to the $x$-direction) is

$$
\mu_{\uparrow}-\mu_{\downarrow}=A_{1} \exp (-x / L)+A_{2} \exp (x / L),
$$

where $A_{1}$ and $A_{2}$ are constants. In contrast the general form of solution to Eq. (1.2) is

$$
n_{\uparrow}-n_{\downarrow}=A_{1} \exp \left(-x / L_{1}\right)+A_{2} \exp \left(-x / L_{2}\right),
$$

where $\lambda_{1}=1 / L_{1}$ and $\lambda_{2}=1 / L_{2}$ are the roots of the quadratic equation,

$$
\lambda^{2}-\lambda \nu E / D-1 / L^{2}=0 .
$$

To understand the physical consequence of the electric field on the spin diffusion, we suppose that a continuous spin imbalance is injected at $x=0,\left.\left(n_{\uparrow}-n_{\downarrow}\right)\right|_{0}$, and the electric field is along the $-x$ direction. The spin polarization will gradually decay in size as the distance from the point of injection increases and eventually go to zero at $\pm \infty$. The distribution of the spin polarization then can be described by

$$
\begin{gathered}
n_{\uparrow}-n_{\downarrow}=\left.\left(n_{\uparrow}-n_{\downarrow}\right)\right|_{0} \exp \left(-x / L_{d}\right), x>0, \\
n_{\uparrow}-n_{\downarrow}=\left.\left(n_{\uparrow}-n_{\downarrow}\right)\right|_{0} \exp \left(x / L_{u}\right), x<0,
\end{gathered}
$$

where we define two quantities $L_{d}$ and $L_{u}$ as the downstream and up-stream spin diffusion lengths, respectively,

$$
L_{d}=\left[-\frac{|e E|}{2} \frac{\nu}{e D}+\sqrt{\left(\frac{|e E|}{2} \frac{\nu}{e D}\right)^{2}+\frac{1}{L^{2}}}\right]^{-1},
$$




$$
L_{u}=\left[\frac{|e E|}{2} \frac{\nu}{e D}+\sqrt{\left(\frac{|e E|}{2} \frac{\nu}{e D}\right)^{2}+\frac{1}{L^{2}}}\right]^{-1}
$$

and $L_{u} L_{d}=L^{2}$. Here $\nu / e D$ in $3 \mathrm{D}$ and $2 \mathrm{D}$ systems can be evaluated via Eqs. 2.12) and (2.13), respectively. For nondegenerate semiconductors, Eqs. (2.22a) and (2.22b) reduce to

$$
\begin{gathered}
L_{d}=\left[-\frac{|e E|}{2 k_{B} T}+\sqrt{\left(\frac{e E}{2 k_{B} T}\right)^{2}+\frac{1}{L^{2}}}\right]^{-1}, \\
L_{u}=\left[\frac{|e E|}{2 k_{B} T}+\sqrt{\left(\frac{e E}{2 k_{B} T}\right)^{2}+\frac{1}{L^{2}}}\right]^{-1} .
\end{gathered}
$$

Equation (2.23a) was reported in Ref. 21.

In the absence of the field, the down-stream and upstream lengths are equal to the intrinsic diffusion length $L$. With increasing field the down-stream diffusion length $L_{d}$ increases, whereas the up-stream diffusion length $L_{u}$ decreases. It was also shown in Ref. 21 that spin transport distance in semiconductors can be increased by an electric field (down-stream diffusion length), but the upstream diffusion length was not discussed. A high-field regime for spin transport in semiconductors can be defined by $E>E_{c}$, where $e E_{c} / k_{B} T=1 / L$. In this regime, $L_{u}$ and $L_{d}$ deviate from $L$ considerably and the spin diffusion behavior is qualitatively different from that in low fields. We emphasize that since $L$ is large in semiconductors, this regime is not beyond realistic fields where most spintronic devices operate. For a typical spin diffusion length in semiconductors, $L=2 \mu \mathrm{m} E_{c}=125 \mathrm{~V} / \mathrm{cm}$ at $T=300 \mathrm{~K}$ and $E_{c}=1.25 \mathrm{~V} / \mathrm{cm}$ at $T=3 \mathrm{~K}$.

The physics of the field effects on the spin diffusion becomes clearer at the strong-field limit, where $|e E| / k_{B} T \gg 1 / L$. In this limit, the electrons move with drift velocity $|E| \nu_{e}$ and so does the spin polarization. $L_{d}$ is simply the distancegver which the carriers move within the spin life time $\tau_{S}, 13,21$

$$
L_{d} \simeq \frac{|e E|}{k_{B} T} L^{2}=\frac{e|E|}{k_{B} T} D \tau_{S}=\nu_{e}|E| \tau_{S} .
$$

For the up-stream diffusion length $L_{u}$ at this limit,

$$
L_{u} \simeq k_{B} T /|e E|
$$

which simply corresponds to a Boltzmann distribution of electrons in a retarding field.13

\section{Carrier densities versus electrochemical potentials}

In the literature of spin transport in metals, the spin polarization is usually described by the splitting of electrochemical potentials for up-spin and down-spin electrons. For nondegenerate semiconductors, the density difference between up-spin and down-spin electrons is a natural way to characterize the spin polarization. It is therefore useful to establish the connection between these two quantities.

The electrochemical potentials for up-spin and downspin electrons in a semiconductor are related to their densities via

$$
n_{\uparrow(\downarrow)}=n_{\uparrow(\downarrow)}^{0}\left[\exp \left(\frac{\mu_{\uparrow(\downarrow)}-\mu_{0}}{k_{B} T}\right)-1\right],
$$

where $\mu_{0}$ is the value that the electrochemical potential would have without spin polarization,

$$
\nabla \mu_{0}=\left(e / \sigma_{s}\right) \mathbf{J}
$$

where $\sigma_{s}$ is the electrical conductivity of the semiconductor and $\mathbf{J}$ is the total electrical current. In a doped semiconductor with a homogeneous carrier concentration, the electrochemical potential $\mu_{0}$ at postion $\mathbf{x}$ is given by

$$
\mu_{0}=\left(e / \sigma_{s}\right) \mathbf{J} \cdot \mathbf{x}-B=e \mathbf{E} \cdot \mathbf{x}-B
$$

where $B$ is a constant. Thus the electrochemical potentials for individual spins are

$$
\mu_{\uparrow(\downarrow)}=k_{B} T \ln \left(1+\frac{n_{\uparrow(\downarrow)}}{n_{\uparrow(\downarrow)}^{0}}\right)+e \mathbf{E} \cdot \mathbf{x}-B .
$$

The electrochemical potential splitting, $\mu_{\uparrow}-\mu_{\downarrow}$, and the density difference, $n_{\uparrow}-n_{\downarrow}$, between up-spin and downspin electrons then are related via

$$
\mu_{\uparrow}-\mu_{\downarrow}=k_{B} T \ln \left[\frac{1+\left(n_{\uparrow}-n_{\downarrow}\right) / 2 n_{\uparrow}^{0}}{1-\left(n_{\uparrow}-n_{\downarrow}\right) / 2 n_{\downarrow}^{0}}\right] .
$$

Therefore it is advantageous to use $n_{\uparrow}-n_{\downarrow}$ instead of $\mu_{\uparrow}-\mu_{\downarrow}$ to describe spin transport in semiconductors, for the spin drift-diffusion equation is linear in terms of the former, but would be nonlinear in terms of the latter.

When $\mu_{\uparrow}-\mu_{\downarrow} \ll k_{B} T$,

$$
\left(n_{\uparrow}-n_{\downarrow}\right)\left(\frac{1}{2 n_{\uparrow}^{0}}+\frac{1}{2 n_{\downarrow}^{0}}\right)=\frac{\mu_{\uparrow}-\mu_{\downarrow}}{k_{B} T},
$$

and we have the drift-diffusion equation for the electrochemical potential splitting,

$$
\nabla^{2}\left(\mu_{\uparrow}-\mu_{\downarrow}\right)+\frac{e \mathbf{E}}{k_{B} T} \cdot \nabla\left(\mu_{\uparrow}-\mu_{\downarrow}\right)-\frac{\mu_{\uparrow}-\mu_{\downarrow}}{L^{2}}=0 .
$$

In this linear differential equation for $\mu_{\uparrow}-\mu_{\downarrow}$, the electric field still plays a central role and there are two distinct diffusion lengths, i.e., the down-stream $\left(L_{d}\right)$ and the upstream $\left(L_{u}\right)$ diffusion lengths, for $\mu_{\uparrow}-\mu_{\downarrow}$. Thus spin transport predicted by Eq. (2.31) would be still qualitatively different from that expected from Eq. (1.1). 


\section{Current versus density spin polarization}

There exist two definitions in literature to characterize spin polarization in nonmagnetic semiconductors. One definition uses the density difference between up-spin and down-spin electrons,

$$
P(x) \equiv \frac{n_{\uparrow}-n_{\downarrow}}{n^{0}},
$$

where $n_{0}=2 n_{\uparrow(\downarrow)}$ is the total electron density. The other uses the current difference between up-spin and downspin electrons,

$$
\alpha(x) \equiv \frac{j_{\uparrow}-j_{\downarrow}}{j_{\uparrow}+j_{\downarrow}}
$$

Generally speaking, these two spin polarization are different, although they are related. To find the relationship between these two polarizations in a homogeneous nonmagnetic semiconductor, we note that

$$
j_{\uparrow}-j_{\downarrow}=e\left(n_{\uparrow}-n_{\downarrow}\right) \nu E+e D \frac{d\left(n_{\uparrow}-n_{\downarrow}\right)}{d x} .
$$

By using the local charge neutrality condition Eq. (2.7), we obtain

$$
\alpha(x)=P(x)+\frac{D}{\nu E} \frac{d P}{d x} .
$$

For a steady spin imbalance injected at $x=0$, as discussed in Sec. II.B, according to the general solution of Eqs. (2.21a and 2.21b,

$$
\begin{array}{lll}
\frac{d\left(n_{\uparrow}-n_{\downarrow}\right)}{d x}=-\frac{1}{L_{d}}\left(n_{\uparrow}-n_{\downarrow}\right), & & x>0, \\
\frac{d\left(n_{\uparrow}-n_{\downarrow}\right)}{d x}=\frac{1}{L_{u}}\left(n_{\uparrow}-n_{\downarrow}\right), & & x<0,
\end{array}
$$

and the relation between the spin polarization of current $\alpha(x)$ and the spin polarization of density $P(x)$ can be written as

$$
\alpha(x)=P(x)\left[1-\left(\frac{\nu}{e D}\right)^{-1} \frac{1}{e E L_{d}}\right]
$$

for $x>0$, and

$$
\alpha(x)=P(x)\left[1+\left(\frac{\nu}{e D}\right)^{-1} \frac{1}{e E L_{u}}\right]
$$

for $x<0 . \nu / e D$ is Eqs. (2.38) and (2.39) can be calculated using Eqs. 2.12) and (2.13) for 3D and 2D systems with different doping concentrations and temperatures. In the nondegenerate limit, Eq. 2.38 reduces to

$$
\alpha(x)=P(x)\left(1-\frac{k_{B} T}{e E L_{d}}\right)
$$

which is equivalent to Eq. (5) in Ref. 21, where the authors studied magnetization $(P)$ in the presence of current with a given spin polarization $(\alpha)$ in semiconductors. In the meantime Eq. 2.39) reduces to

$$
\alpha(x)=P(x)\left(1+\frac{k_{B} T}{e E L_{u}}\right) .
$$

Thus in semiconductors $\alpha(x)$ is proportional to $P(x)$, and the ratio between them depends on the electric field and its direction.

\section{FIELD ENHANCED SPIN INJECTION IN FM/NS STRUCTURES}

We first consider a simple one-dimensional spin injection structure to elucidate the underlying physics of electric-field enhanced spin injection. This injection structure comprises a semi-infinite degenerate ferromagnet $(x<0)$ and a semi-infinite nonmagnetic nondegenerate semiconductor $(x>0)$. Electrons are injected from the magnet into the semiconductor, and therefore, the electric field is antiparallel to the $x$-axis. In the ferromagnet the electrochemical potentials for individual spins satisfy Eq. (2.16), which has the following general solution:

$$
\frac{1}{e J}\left(\begin{array}{c}
\mu_{\uparrow} \\
\mu_{\downarrow}
\end{array}\right)=\frac{x}{\sigma_{\uparrow}^{f}+\sigma_{\downarrow}^{f}}\left(\begin{array}{c}
1 \\
1
\end{array}\right)+C\left(\begin{array}{c}
1 / \sigma_{\uparrow}^{f} \\
-1 / \sigma_{\downarrow}^{f}
\end{array}\right) e^{x / L^{(f)}},
$$

where $\sigma_{\uparrow(\downarrow)}^{f}$ is the up-spin (down-spin) electrical conductivity of the ferromagnet, and $J$ is the total electron current, which is a constant throughout the structure in steady state. We use $L^{(f)}$ and $L^{(s)}$ to denote the intrinsic spin diffusion length in the ferromagnet and in the semiconductor, respectively.

In the semiconductor, up-spin and down-spin electron densties satisfy the spin drift-diffusion equation for nondegenerate systems, Eq. (1.2), as well as the local charge neutrality condition, Eq. (2.7). The general solution can be written as

$$
n_{\uparrow(\downarrow)}=+(-) A \exp \left(-x / L_{d}\right),
$$

and accordingly the electrochemical potentials for individual spins are

$$
\mu_{\uparrow(\downarrow)}=k_{B} T \ln \left[1+(-) \frac{2 A e^{-x / L_{d}}}{n_{0}}\right]+e E x-B .
$$

Here $n_{0}$ is total electron density.

In general, a Schottky barrier will form between the magnet and the semiconductor when the two materials are placed together. Since the charge neutrality condition would be severely violated in the depletion region of a Schottky barrier, a wide depletion region is undesirable for spin transport and spin coherence. Specifically the 
presence of holes dramatically shortens the electron spin coherence time. We consider instead structures with a very thin interfacial barrier between the magnet and the semiconductor. A spin-selective interfacial barrier was examined in Refs. 2, 4,5,6 as a way to circumvent the resistance mismatch obstacle for spin injection from a ferromagnetic metal into a semiconductor. If there is no spin-flip scattering at the interface, the current for an individual spin is continuous across the interface and is related to the spin-dependent electrochemical potential change across the interface via Ohmic's law, giving rise to the following boundary conditions:

$$
\begin{aligned}
& j_{\uparrow}\left(0^{-}\right)=G_{\uparrow}\left[\mu_{\uparrow}\left(0^{+}\right)-\mu_{\uparrow}\left(0^{-}\right)\right], \\
& j_{\downarrow}\left(0^{-}\right)=G_{\downarrow}\left[\mu_{\downarrow}\left(0^{+}\right)-\mu_{\downarrow}\left(0^{-}\right)\right], \\
& j_{\uparrow}\left(0^{-}\right)-j_{\downarrow}\left(0^{-}\right)=j_{\uparrow}\left(0^{+}\right)-j_{\downarrow}\left(0^{+}\right),
\end{aligned}
$$

where $G_{\uparrow(\downarrow)}$ is the interfacial conductance for up-spin (down-spin) electrons and the current of individual spins $j_{\uparrow(\downarrow)}$ can be calculated via $e j_{\uparrow(\downarrow)}=\sigma_{\uparrow(\downarrow)} d \mu_{\uparrow(\downarrow)} / d x$. These three equations completely determine the three unknown coefficients $A, B, C$ in Eqs. (3.1)-(3.3).

The solution of $n_{\uparrow(\downarrow)}$ in Eq. (3.2) and the relation of Eq. (2.40) indicate that in the semiconductor $\alpha(x)=$ $\alpha_{0} e^{-x / L_{d}}$, where $\alpha_{0}$ is the spin injection efficiency at the interface. We obtain an equation for $\alpha_{0}$,

$$
\frac{G_{\uparrow}^{-1}-G_{\downarrow}^{-1}}{2}+\frac{p_{f}\left(G_{\uparrow}^{-1}+G_{\downarrow}^{-1}\right)}{2}+\left(\alpha_{0}-p_{f}\right)\left[\frac{G_{\uparrow}^{-1}+G_{\downarrow}^{-1}}{2}+\frac{2 L^{(f)}}{\left(1-p_{f}^{2}\right) \sigma_{f}}\right]=\frac{k_{B} T}{e E \sigma_{s}} \ln \frac{-k_{B} T / e E L_{u}+\alpha_{0}}{-k_{B} T / e E L_{u}-\alpha_{0}},
$$

where $\sigma_{f}=\sigma_{\uparrow}^{f}+\sigma_{\downarrow}^{f}$ is the conductivity of the ferromagnet, $\sigma_{s}=n_{0} e \nu$ the conductivity of the semiconductor, and $p_{f}=\left(\sigma_{\uparrow}^{f}-\sigma_{\downarrow}^{f}\right) / \sigma_{f}$ the spin polarization in the ferromagnet.

We solve this equation and plot the spin injection of current $\alpha_{0}$ as a function of the electric field in Fig. 2. We see that the electric field can substantially enhance the spin injection efficiency in FM/NS structures. We note that spin injection enhancement from a spinselective interfacial barrier between the ferromagnet and the semicondistor, which has been identified in the lowfield regime, 156 becomes more pronounced in the highfield regime.

In the small spin polarization limit, $n_{\uparrow(\downarrow)} / n_{0} \ll 1, \alpha(x)$ can be expressed in an explicit form,

$$
\begin{aligned}
\alpha(x) & =\left[\frac{L^{(f)}}{\left(1-p_{f}^{2}\right) \sigma_{f}}+\frac{L_{u}}{\sigma_{s}}+\frac{G_{\uparrow}+G_{\downarrow}}{4 G_{\uparrow} G_{\downarrow}}\right]^{-1} \\
& \times\left[\frac{p_{f} L^{(f)}}{\left(1-p_{f}^{2}\right) \sigma_{f}}+\frac{G_{\uparrow}-G_{\downarrow}}{4 G_{\uparrow} G_{\downarrow}}\right] e^{-x / L_{d}} .
\end{aligned}
$$

This expression clearly shows that the electric field and the spin-selective interfacial resistance both enhance spin injection, but in different ways. The electric-field effects on spin injection can be described in terms of the two field-induced diffusion lengths. Both diffusion lengths affect spin injection favorably: The up-stream length $L_{u}$ controls the relevant resistance in the semiconductor, which determines the spin injection efficiency. With increasing field this effective resistance, $L_{u} / \sigma_{s}$, becomes smaller, and accordingly the spin injection efficiency is enhanced. The transport distance of the injected spin

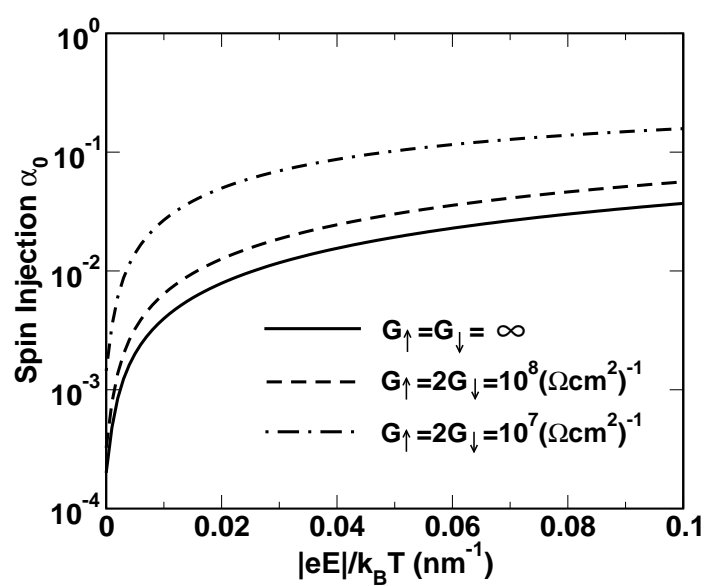

FIG. 2: Spin injection efficiency $\alpha_{0}$ as a function of electric field. The interfacial conductance for up-spin electrons is twice larger than that for down-spin electrons, $G_{\uparrow}=2 G_{\downarrow}$. Solid, dashed, and dot-dashed lines correspond to $G_{\uparrow}=\infty$ (transparent interface), $10^{8}$, and $10^{7}\left(\Omega \mathrm{cm}^{2}\right)^{-1}$, respectively. Other parameters are $p_{f}=0.5, L^{(f)}=60 \mathrm{~nm}, L^{(s)}=2 \mu \mathrm{m}$, and $\sigma_{f}=100 \sigma_{s}=10^{3}(\Omega \mathrm{cm})^{-1}$.

polarization in the semiconductor, however, is controlled by the down-stream length $L_{d}$. As the field increases, this distance becomes longer. On the other hand, the spin-selective interfacial barrier provides another spin polarization source besides the spin-aligned electrons in the ferromagnet and acts as a spin filter which permits electrons with a particular spin to pass through the inter- 
face, and therefore enhances spin injection. Moreover, the spin-selective barrier has no effect on the transport distance of the injected spin polarization in the semiconductor.

We now contrast for (3.6) with that obtained by previous calculations 3 日e.6 based on Eq. (1.1). The spin injection

$$
\begin{aligned}
\alpha(x) & =\left[\frac{L^{(f)}}{\left(1-p_{f}^{2}\right) \sigma_{f}}+\frac{L^{(s)}}{\sigma_{s}}+\frac{G_{\uparrow}+G_{\downarrow}}{4 G_{\uparrow} G_{\downarrow}}\right]^{-1} \\
& \times\left[\frac{p_{f} L^{(f)}}{\left(1-p_{f}^{2}\right) \sigma_{f}}+\frac{G_{\uparrow}-G_{\downarrow}}{4 G_{\uparrow} G_{\downarrow}}\right] e^{-x / L^{(s)}}
\end{aligned}
$$

is given by the zero-field result of Eq. (3.6). For a transparent interface with $G_{\uparrow}^{-1}=G_{\downarrow}^{-1}=0$, the effective resistance in the magnet, $L^{(f)} / \sigma_{f}$, is much less than its counterpart in the semiconductor, $L^{(s)} / \sigma_{s}$ (as $L^{(f)} \ll L^{(s)}$ and $\sigma_{f} \gg \sigma_{s}$ ). Thus Eq. (3.7) suggests that this resistance mismatch makes it virtually impossible to realize an appreciable spin injection from a ferromagnetic metal to a semiconductor without spin-selective interfacial barrier. However, the more general description of the spin transport in semiconductors indicates that the effective semiconductor resistance to be compared with $L^{(f)} / \sigma_{f}$ should be $L_{u} / \sigma_{s}$ rather than $L^{(s)} / \sigma_{s}$. Since $L_{u}$ can be smaller than $L^{(s)}$ by orders of magnitude in the highfield regime, this "conductivity mismatch" obstacle may be overcome with the help of strong electric fields, or equivalently, large injection currents. We note that although it has been realized that spin injection can be enhanced by increasing the total injection current, the treatment there used Eq. (1.1) to describe spin transport in nondegenerate semiconductors, where the electric-field effects were not taken into account. Thus the physics of the field-dependent spin transport was not captured and the treatment was incomplete.

In the presence of the interfacial barrier, the relative importance of the two mechanisms for the spin injection efficiency enhancement, electric field and spin-selective interfacial barrier, depends on the relative magnitude of $R_{f}=L^{(f)} /\left(1-p_{f}^{2}\right) \sigma_{f}, R_{s} \equiv L^{(s)} / \sigma_{s}$, and $R_{i} \equiv$ $\left(G_{\uparrow}+G_{\downarrow}\right) / 4 G_{\uparrow} G_{\downarrow}$, the resistances of the ferromagnet, the semiconductor, and the interface, which may vary from system to system. For systems with $R_{i} \gg R_{s} \gg R_{f}$, the spin-selective interfacial barrier dominates in enhancing the spin injection efficiency. On the other hand, for systems with $R_{s} \gg R_{i} \gg R_{f}$ or $R_{s} \gg R_{f} \gg R_{i}$, the electric field can enhance spin injection efficiently. The transport distance of injected spin polarization in the semiconductor, however, can only be enhanced by the electric field, even in systems with $R_{i} \gg R_{s} \gg R_{f}$. Furthermore, the two mechanisms have different temperature and field dependences. The field-enhanced spin injection efficiency increases with the electric field (current) and decreases with the temperature. The interface-enhanced spin injection likely depends on neither the field nor the temperature.
To quantitatively demonstrate the electric-field effects on spin injection, we choose the realistic parameters of a spin injection device as follows: $p_{f}=0.5$ and $L^{(f)}=60$ $\mathrm{nm}$ (as in Co) $22 L^{(s)}=2 \mu \mathrm{m}$ (as in GaAs). First we examine the structures without an interfacial barrier. For a ferromagmetic metal/semiconductor structure, e.g., $\mathrm{Co} / \mathrm{GaAs}, \sigma_{f} \simeq 10^{4} \sigma_{s}$, the spin injection efficiency increases from $2 \times 10^{-6}$ at zero field to $2 \%$ at $|e E| / k_{B} T=5$ $\mathrm{nm}^{-1}$. For a ferromagnetic semiconductor with $p_{f} \sim 0.5$ and $\sigma_{f} \sim 100 \sigma_{s}$, the spin injection efficiency increases from $0.02 \%$ at zero field to $2 \%$ at $|e E| / k_{B} T=0.05 \mathrm{~nm}^{-1}$, which corresponds to $|E|=125 \mathrm{~V} / \mathrm{cm}$, or $|J|=1250$ $\mathrm{A} / \mathrm{cm}^{2}$ for a typical semiconductor conductivity $\sigma_{s}=10$ $(\Omega \mathrm{cm})^{-1}$, at $T=3 \mathrm{~K}$.

Suppose there existed a spin-selective interfacial barrier between the magnet and the semiconductor with $G_{\uparrow}=2 G_{\downarrow}=10^{7}\left(\Omega \mathrm{cm}^{2}\right)^{-1}$. For the Co/GaAs structure, the spin injection efficiency would be $0.1 \%$ at zero field (much greater than $2 \times 10^{-6}$ in a similar injection structure without a spin-selective interfacial barrier), which can be further enhanced to $10 \%$ at $|e E| / k_{B} T=0.06$ $\mathrm{nm}^{-1}$, or $E=15 \mathrm{kV} / \mathrm{cm}$ at $T=300 \mathrm{~K}$. For the spin injection structure from the ferromagnetic semiconductor, the spin injection efficiency is $0.1 \%$ at zero field (also considerably greater than $0.02 \%$ in a similar structure without a spin-selective interfacial barrier), which can be further increased to $10 \%$ at $|e E| / k_{B} T=0.05 \mathrm{~nm}^{-1}$. Thus the combination of electric field and spin-selective interfacial barrier may help explain the large spin injection percentages from ZnMnSe to ZnSe 2324 from GaMnAs to GaAs, 25 from Fe to GaAs, 262728 as well as the dramatic increase in spin injection with current in Ref. 27.

Figure 3 shows spin polarization of current $\left(\alpha_{0}\right)$ and spin polarization of density $\left(P_{0}\right)$ at the interface as a function of electric field. According to Eq. (2.40), $P_{0}=\alpha_{0}\left(|e E| L_{u} / k_{B} T\right)$. We can see that in the low-field regime, the density polarization in the semiconductor is much smaller than the current polarization; whereas in the high-field regime, the two polarizations become equal. Thus the difference between high-field injection and lowfield injection is that a strong electric field in high-field injection results in a macroscopic density difference between up-spin and down-spin electrons; whereas in lowfield injection the carrier densities of up-spin and down spin electrons remain the same.

Another important quantity is the boundary resistance $R_{b}$, which can be used to analyze how easy a current can convert its spin while flowing from the magnet into the semiconductor,

$$
R_{b}=\frac{\mu_{0}\left(0^{+}\right)-\mu_{0}\left(0^{-}\right)}{e J}
$$

which can be expressed in terms of the spin injection efficiency and the resistances of the magnet, the semi- 


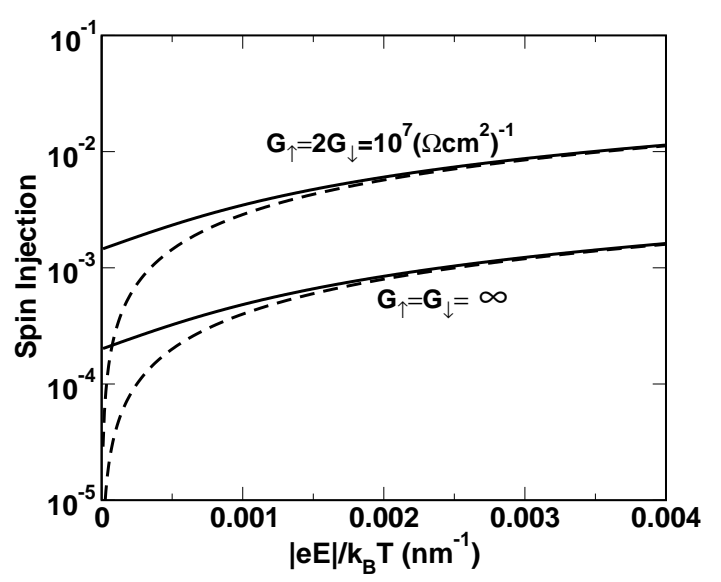

FIG. 3: Spin injection as a function of electric field. Solid and dashed lines describe spin polarization of current $\alpha_{0}$ and spin polarization of density $P_{0}$ at the interface, respectively. The lower curves are for transparent interface structure and the upper ones are for structure with a spin selective interfacial barrier, $G_{\uparrow}=2 G_{\downarrow}=10^{7}\left(\Omega \mathrm{cm}^{2}\right)^{-1}$. Other parameters are the same as in Fig. 2.

conductor, and the interface.

$$
\begin{aligned}
R_{b} & =\frac{-B}{e J}=\left(p_{f}-\alpha_{0}\right)\left[\frac{p_{f} L^{(f)}}{\left(1-p_{f}^{2}\right) \sigma_{f}}+\frac{G_{\uparrow}-G_{\downarrow}}{4 G_{\uparrow} G_{\downarrow}}\right] \\
& +\frac{G_{\uparrow}+G_{\downarrow}}{4 G_{\uparrow} G_{\downarrow}}-p_{f} \frac{G_{\uparrow}-G_{\downarrow}}{4 G_{\uparrow} G_{\downarrow}} .
\end{aligned}
$$

We plot $R_{b}$ in Fig. 4 as a function of electric field. With increasing field, the boundary resistance decreases, indicating that the field helps current conversion from a higher polarization (in the magnet) to a lower polarization (in the semiconductor). For structures with a transparent interface, in the small polarization limit $\left[n_{\uparrow(\downarrow)} / n_{0} \ll 1\right]$,

$$
R_{b}=\left[\frac{L^{(f)}}{\sigma_{f}}+\frac{L_{u}}{\sigma_{s}}\left(1-p_{f}^{2}\right)\right]^{-1} p_{f}^{2} \frac{L^{(f)}}{\sigma_{f}} \frac{L_{u}}{\sigma_{s}},
$$

which clearly shows that the boundary resistance is determined by the up-stream diffusion length $L_{u}$ rather than $L^{(s)}$ and should be a function of electric field.

\section{SPIN INJECTION IN FM/NS/FM STRUCTURES}

In this section we consider a sandwiched structure that comprises a semi-infinite ferromagnet $(x<0)$, a nonmagnetic semiconductor with width $x_{0}$, and a semiinfinite ferromagnet $\left(x>x_{0}\right) . x_{0}$ is assumed to be much shorter than the semiconductor intrinsic spin diffusion length $L^{(s)}$, as in most spintronic devices. The two ferromagnets are otherwise identical with possible different

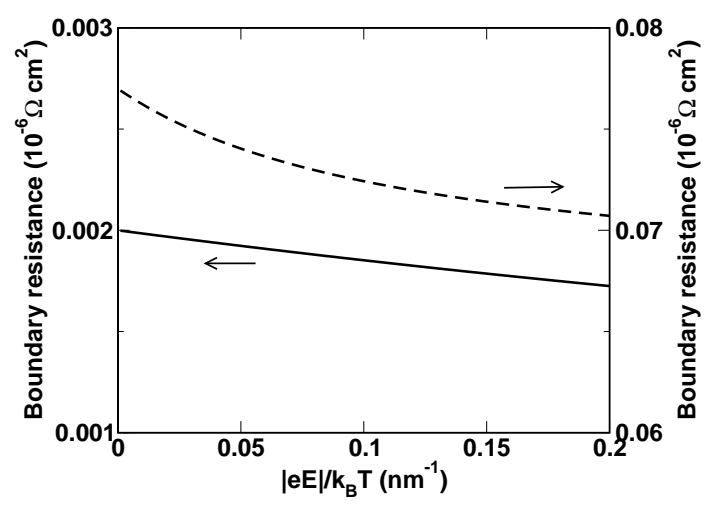

FIG. 4: Boundary resistance $R_{b}$ as a function of electric field. Solid line is for structure with transparent interface. Dashed line is for structure with a spin-selective interfacial barrier, $G_{\uparrow}=2 G_{\downarrow}=10^{7}\left(\Omega \mathrm{cm}^{2}\right)^{-1}$. Other parameters are the same as in Fig. 2.

orientations of magnetization (parallel and anti-parallel). Electrons are injected from the left magnet to the semiconductor and the electric field is antiparallel to the $x$ axis. The spin transport in the magnets is described by Eq. (2.16) with the following general solutions:

$$
\frac{1}{e J}\left(\begin{array}{c}
\mu_{\uparrow} \\
\mu_{\downarrow}
\end{array}\right)=\frac{x}{\sigma_{\uparrow}^{L}+\sigma_{\downarrow}^{L}}\left(\begin{array}{c}
1 \\
1
\end{array}\right)+C_{L}\left(\begin{array}{c}
1 / \sigma_{\uparrow}^{L} \\
-1 / \sigma_{\downarrow}^{L}
\end{array}\right) e^{x / L^{(f)}}
$$

in the left magnet $(x<0)$, and

$$
\begin{aligned}
\frac{1}{e J}\left(\begin{array}{c}
\mu_{\uparrow} \\
\mu_{\downarrow}
\end{array}\right) & =\left(\frac{x}{\sigma_{\uparrow}^{R}+\sigma_{\downarrow}^{R}}+B_{1}\right)\left(\begin{array}{c}
1 \\
1
\end{array}\right) \\
& +C_{R}\left(\begin{array}{c}
1 / \sigma_{\uparrow}^{R} \\
-1 / \sigma_{\downarrow}^{R}
\end{array}\right) e^{-\left(x-x_{0}\right) / L^{(f)}}
\end{aligned}
$$

in the right magnet $\left(x>x_{0}\right)$. Here we use superscript or subscript $L(R)$ to label the quantities in the left (right) ferromagnet.

The spin-dependent electron densities in the semiconductor satisfy Eqs. (1.2) and (2.7), and the general solutions at $0<x<x_{0}$ can be written as

$$
n_{\uparrow(\downarrow)}=+(-)\left[A_{0} e^{-x / L_{d}}+A_{1} e^{\left(x-x_{0}\right) / L_{u}}\right],
$$

and therefore the electrochemical potentials for individual spins are

$$
\begin{aligned}
\mu_{\uparrow(\downarrow)} & =k_{B} T \ln \left[1+(-) \frac{2 A_{0} e^{-x / L_{d}}+2 A_{1} e^{\left(x-x_{0}\right) / L_{u}}}{n_{0}}\right] \\
& +e E x-B_{0} .
\end{aligned}
$$

The six unknowns in the above solutions, $A_{0}, A_{1}, B_{0}$, $B_{1}, C_{L}$, and $C_{R}$, are determined by the following six 
independent boundary conditions:

$$
\begin{aligned}
& j_{\uparrow}\left(0^{-}\right)=G_{\uparrow}\left[\mu_{\uparrow}\left(0^{+}\right)-\mu_{\downarrow}\left(0^{-}\right)\right], \\
& j_{\downarrow}\left(0^{-}\right)=G_{\downarrow}\left[\mu_{\downarrow}\left(0^{+}\right)-\mu_{\downarrow}\left(0^{-}\right)\right], \\
& j_{\uparrow}\left(0^{-}\right)-j_{\downarrow}\left(0^{-}\right)=j_{\uparrow}\left(0^{+}\right)-j_{\downarrow}\left(0^{+}\right), \\
& j_{\uparrow}\left(x_{0}^{+}\right)=\tilde{G}_{\uparrow}\left[\mu_{\uparrow}\left(x_{0}^{+}\right)-\mu_{\downarrow}\left(x_{0}^{-}\right)\right], \\
& j_{\downarrow}\left(x_{0}^{+}\right)=\tilde{G}_{\downarrow}\left[\mu_{\downarrow}\left(x_{0}^{+}\right)-\mu_{\downarrow}\left(x_{0}^{-}\right)\right], \\
& j_{\uparrow}\left(x_{0}^{-}\right)-j_{\downarrow}\left(x_{0}^{-}\right)=j_{\uparrow}\left(x_{0}^{+}\right)-j_{\downarrow}\left(x_{0}^{+}\right),
\end{aligned}
$$

where $G_{\uparrow(\downarrow)}$ and $\tilde{G}_{\uparrow(\downarrow)}$ are the spin-dependent conductances at the left and the right interfaces, respectively.

We find the two equations for the spin polarizations of current at interfaces, $\alpha(0)$ and $\alpha\left(x_{0}\right)$, by matching the above boundary conditions, Eqs. (4.5a-f),

$$
\left\{\begin{array}{c}
\frac{p_{L}\left(G_{\uparrow}+G_{\downarrow}\right)}{2 G_{\uparrow} G_{\downarrow}}-\frac{G_{\uparrow}-G_{\downarrow}}{2 G_{\uparrow} G_{\downarrow}}+\left[\alpha(0)-p_{L}\right]\left[\frac{G_{\uparrow}+G_{\downarrow}}{2 G_{\uparrow} G_{\downarrow}}+\frac{2 L^{(f)}}{\left(1-p_{L}^{2}\right) \sigma_{f}}\right]=\frac{k_{B} T}{e E \sigma_{s}} \ln \frac{1+z_{1}}{1-z_{1}} \\
\frac{p_{R}\left(\tilde{G}_{\uparrow}+\tilde{G}_{\downarrow}\right)}{2 \tilde{G}_{\uparrow} \tilde{G}_{\downarrow}}-\frac{\tilde{G}_{\uparrow}-\tilde{G}_{\downarrow}}{2 \tilde{G}_{\uparrow} \tilde{G}_{\downarrow}}+\left[\alpha\left(x_{0}\right)-p_{R}\right]\left[\frac{\tilde{G}_{\uparrow}+\tilde{G}_{\downarrow}}{2 \tilde{G}_{\uparrow} \tilde{G}_{\downarrow}}+\frac{2 L^{(f)}}{\left(1-p_{R}^{2}\right) \sigma_{f}}\right]=\frac{k_{B} T}{e E \sigma_{s}} \ln \frac{1-z_{2}}{1+z_{2}}
\end{array}\right.
$$

where $p_{L}$ and $p_{R}$ are the spin polarization in the left and right magnets, respectively, and

$$
\begin{aligned}
& z_{1}=\frac{e E}{k_{B} T} \frac{\alpha\left(x_{0}\right)\left(L_{u}+L_{d}\right)-\alpha(0)\left(L_{u} e^{x_{0} / L_{u}}+L_{d} e^{-x_{0} / L_{d}}\right)}{e^{x_{0} / L_{u}}-e^{-x_{0} / L_{d}}}, \\
& z_{2}=\frac{e E}{k_{B} T} \frac{\alpha\left(x_{0}\right)\left(L_{u} e^{-x_{0} / L_{u}}+L_{d} e^{x_{0} / L_{d}}\right)-\alpha(0)\left(L_{u}+L_{d}\right)}{e^{x_{0} / L_{d}}-e^{-x_{0} / L_{u}}} .
\end{aligned}
$$

When $\alpha(0)$ and $\alpha\left(x_{0}\right)$ are known, we can express the spin polarization of current in the semiconductor $(0<$ $\left.x<x_{0}\right)$ as

$$
\begin{aligned}
\alpha(x) & =\alpha(0) \frac{e^{-\left(x-x_{0}\right) / L_{d}}-e^{\left(x-x_{0}\right) / L_{u}}}{e^{x_{0} / L_{d}}-e^{-x_{0} / L_{u}}} \\
& -\alpha\left(x_{0}\right) \frac{e^{-x / L_{d}}-e^{x / L_{u}}}{e^{x_{0} / L_{u}}-e^{-x_{0} / L_{d}}},
\end{aligned}
$$

and the spin polarization of density in the semiconductor $\left(0<x<x_{0}\right)$ as

$$
\begin{aligned}
P(x) & =-\alpha(0) \frac{e E}{k_{B} T} \frac{L_{u} e^{-\left(x-x_{0}\right) / L_{d}}+L_{d} e^{\left(x-x_{0}\right) / L_{u}}}{e^{x_{0} / L_{d}}-e^{-x_{0} / L_{u}}} \\
& +\alpha\left(x_{0}\right) \frac{e E}{k_{B} T} \frac{L_{u} e^{-x / L_{d}}+L_{d} e^{x / L_{u}}}{e^{x_{0} / L_{u}}-e^{-x_{0} / L_{d}}}
\end{aligned}
$$

Figure 5 depicts the spin injection efficiency at the left interface $\left(\alpha_{0}\right)$ as a function of electric field for the parallel configuration $p_{L}=p_{R}=p_{f}$ and the anti-parallel configuration $p_{L}=-p_{R}=p_{f}$. For structures with transparent interfaces $\left(G_{\uparrow}^{-1}=G_{\downarrow}^{-1}=\tilde{G}_{\uparrow}^{-1}=\tilde{G}_{\downarrow}^{-1}=0\right)$, we see that the spin injection in both configurations can be enhanced by orders of magnitude by increasing the field. In the low-field regime, spin injection can only be achieved in structures with the parallel configuration. In the highfield regime, spin injection from the left magnet into the semiconductor for both configurations are the same, indicating that only the magnet from which carriers are injected is important to the spin injection efficiency.
For structures with transparent interfaces, spin polarization in the semiconductor $\alpha(x)$ can be expressed in compact forms in both the low- and high-field regimes. In fact, in the low-field regime, where $x_{0} \ll L_{u}, L_{d}$, we reproduce Eq. (7) in Ref. 3,

$$
\alpha(x)=\alpha=\left[\frac{2 L^{(f)}}{\left(1-p_{f}^{2}\right) \sigma_{f}}+\frac{x_{0}}{\sigma_{s}}\right]^{-1} \frac{\left(p_{L}+p_{R}\right) L^{(f)}}{\left(1-p_{f}^{2}\right) \sigma_{f}}
$$

for $0<x<x_{0}$. The above expression indicates that the spin injection strongly depends on the relative orientation of the two ferromagnetic metals: For the parallel configuration, $\alpha(x)$ is finite; whereas for the antiparallel configuration, spin injection is not possible. In this limit, the left magnet and the right one are equally important in determining spin injection. The magnitude of spin injection $\alpha$ is determined by the ratio of the effective resistances in the magnet $\left(L^{(f)} / \sigma_{f}\right)$ and in the semiconductor $\left(x_{0} / \sigma_{s}\right)$. For typical device parameters $L^{(f)} / \sigma_{f} \ll x_{0} / \sigma_{s}$, and the spin injection efficiency would be extremely small due to this resistance mismatch. The spin polarization of density in this limit can be written as $P(x)=-\alpha\left(\frac{e E}{k_{B} T}\right)^{2} L_{u} L_{d}$. At the zero-field limit, the density difference between up-spin and down-spin electrons vanishes.

On the other hand, in the high-field regime, $L_{u}$ and $L_{d}$ can differ by orders of magnitude, and usually $L_{u} \ll$ 
$x_{0} \ll L_{d}$. We find that in this limit, for $0<x<x_{0}$,

$$
\alpha(x)=\alpha=\left[\frac{L^{(f)}}{\left(1-p_{f}^{2}\right) \sigma_{f}}+\frac{L_{u}}{\sigma_{s}}\right]^{-1} \frac{p_{L} L^{(f)}}{\left(1-p_{L}^{2}\right) \sigma_{f}},
$$

and $P(x)=\alpha|e E| L_{u} / k_{B} T$, which is close to $\alpha$ in the strong-field limit. We see that in the high-field regime, spin injection is locally determined by the magnet from which carriers are injected, and the "remote" magnet that collects the current becomes irrelevant in determining the spin injection efficiency. Moreover, the effective resistance of the semiconductor to be compared with that of the magnet $\left(L^{(f)} / \sigma_{f}\right)$ is $L_{u} / \sigma_{s}$ rather than $x_{0} / \sigma_{s}$. In this regime, the spin polarization of current and the spin polarization of density have similar amplitude. Thus in the high-field regime, according to Eq. (4.11), the spin injection behavior in the sandwiched FM/NS/FM structure would be the same as in a simpler FM/NS structure. The reason that the second magnet becomes unimportant for spin injection in the high-field regime is that the influence of the second magnet on spin transport in the semiconductor is localized within the up-stream length $\left(L_{u}\right)$ from the magnet. $L_{u}$ decreases with increasing field and would be much shorter than $x_{0}$ in the high-field regime.

In Fig. 5, we also plot spin injection as a function of the electric field in structure with two identical spinselective interfacial barriers between the magnet and the semiconductor. In the calculations it is assumed that $G_{\uparrow(\downarrow)}=\tilde{G}_{\uparrow(\downarrow)} \neq 0$ for the parallel configuration, and $G_{\uparrow(\downarrow)}=\tilde{G}_{\downarrow(\uparrow)} \neq 0$ for the anti-parallel configuration. We see that the electric field substantially enhances spin injection in structures with spin-selective interfacial barrier. In the high-field regime, as in the transparent case, spin injection from the left magnet into the semiconductor does not depend on the orientation of the right magnet.

\section{SPIN INJECTION IN FM/NS/NS STRUCTURES}

In semiconductor spin injection structures, a highly doped nonmagnetic semiconductor $\left(\mathrm{NS}^{+}\right)$is often placed near the magnet interface to overcome the Schottky barrier between a magnet and a semiconductor. This configuration is also intrinsic to FM/InAs, where densely occupied surface states form at the interface. Here we consider an injection structure that comprises a semiinfinite magnet $(x<0)$, a finite nonmagnetic semiconductor with conductivity $\sigma_{s}$ and carrier concentration $n_{0}$ $\left(0<x<x_{0}\right)$, and a semi-infinite nonmagnetic semiconductor with conductivity $\tilde{\sigma}_{s}$ and carrier concentration $\tilde{n}_{0}$ $\left(x>x_{0}\right)$. The electrochemical potentials in the magnet satisfy Eq. (2.16), and the electron densities in semiconductors satisfy Eqs. (1.2) and (2.7). The general solution of the electrochemical potentials in the magnet and the

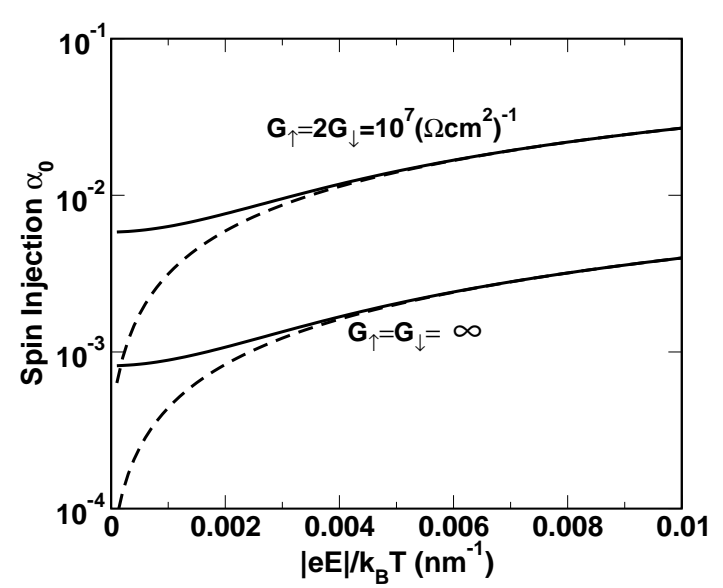

FIG. 5: Spin injection efficiency $\alpha_{0}$ as a function of electric field. Solid and dashed lines correspond to parallel configuration $\left(p_{L}=p_{R}=p_{f}\right)$ and anti-parallel configuration $\left(p_{L}=\right.$ $\left.-p_{R}=p_{f}\right)$, respectively. The lower curves are for structure with transparent interface. The upper curves are for structure with a spin-selective interfacial barrier, $G_{\uparrow}=2 G_{\downarrow}=10^{7}(\Omega$ $\left.\mathrm{cm}^{2}\right)^{-1}$. Other parameters are $p_{f}=0.5, L^{(f)}=60 \mathrm{~nm}$, $x_{0}=1 \mu \mathrm{m}, L^{(s)}=2 \mu \mathrm{m}$, and $\sigma_{f}=100 \sigma_{s}=10^{3}(\Omega \mathrm{cm})^{-1}$.

semiconductors can be written as

$$
\frac{1}{e J}\left(\begin{array}{c}
\mu_{\uparrow} \\
\mu_{\downarrow}
\end{array}\right)=\frac{x}{\sigma_{\uparrow}^{f}+\sigma_{\downarrow}^{f}}\left(\begin{array}{c}
1 \\
1
\end{array}\right)+C\left(\begin{array}{c}
1 / \sigma_{\uparrow}^{f} \\
-1 / \sigma_{\downarrow}^{f}
\end{array}\right) e^{x / L^{(f)}}
$$

for $x<0$,

$$
\begin{aligned}
\mu_{\uparrow(\downarrow)} & =k_{B} T \ln \left[1+(-) \frac{2 A_{0} e^{-x / L_{d}}+2 A_{1} e^{\left.\left(x-x_{0}\right) / L_{u}\right)}}{n_{0}}\right] \\
& +\frac{e J x}{\sigma_{s}}-B_{0}
\end{aligned}
$$

for $0<x<x_{0}$, and

$$
\mu_{\uparrow(\downarrow)}=k_{B} T \ln \left[1+(-) \frac{2 A_{2} e^{-\left(x-x_{0}\right) / \tilde{L}_{d}}}{\tilde{n}_{0}}\right]+\frac{e J x}{\tilde{\sigma}_{s}}-B_{1}
$$

for $x>x_{0}$.

Here we consider structures without interface resistance between two semiconductor regions, and the boundary conditions are given by Eq. (4.5a-f) with $\tilde{G}_{\uparrow}^{-1}=\tilde{G}_{\downarrow}^{-1}=0$. These equations completely determine the six unknowns, $A_{i}(i=0,1,2), B_{i}(i=1,2)$, and $C$, in expressions of Eqs. (5.1)-(5.3).

We obatin an equation for $z=\alpha\left(x_{0}\right)$, the spin polarization of current at the interface between the two semiconductors, 


$$
\begin{aligned}
\frac{G_{\uparrow}^{-1}-G_{\downarrow}^{-1}}{2}+\frac{p_{f}\left(G_{\uparrow}^{-1}+G_{\downarrow}^{-1}\right)}{2} & -\frac{k_{B} T}{e J} \ln \frac{-k_{B} T \tilde{\sigma}_{s} / e J \tilde{L}_{u}+(a+b) z}{-k_{B} T \tilde{\sigma}_{s} / e J \tilde{L}_{u}-(a+b) z} \\
& =\left[\frac{2 L^{(f)}}{\left(1-p_{L}^{2}\right) \sigma_{f}}+\frac{G_{\uparrow}^{-1}+G_{\downarrow}^{-1}}{2}\right]\left[\left(\frac{\tilde{L}_{u} \sigma_{s}}{L_{u} \tilde{\sigma}_{s}} a-\frac{\tilde{L}_{u} \sigma_{s}}{L_{d} \tilde{\sigma}_{s}} b\right) z-p_{f}\right]
\end{aligned}
$$

where $J=\sigma_{s} E=\tilde{\sigma}_{s} \tilde{E}$, and

$$
\begin{aligned}
& a=\left[\sigma_{s}\left(\frac{1}{L_{d}}+\frac{1}{L_{u}}\right)\right]^{-1}\left(\frac{\tilde{\sigma}_{s}}{\tilde{L}_{d}}+\frac{\sigma_{s}}{L_{u}}\right) e^{x_{0} / L_{d}}, \\
& b=\left[\sigma_{s}\left(\frac{1}{L_{d}}+\frac{1}{L_{u}}\right)\right]^{-1}\left(\frac{-\tilde{\sigma}_{s}}{\tilde{L}_{d}}+\frac{\sigma_{s}}{L_{d}}\right) e^{-x_{0} / L_{u}} .
\end{aligned}
$$

Figure 6 illustrates the spin injection efficiency $\alpha\left(x_{0}\right)$ as a function of the total electric current $J$. We see that in the low-field regime, the conductivities of both semi- conductors are important to determine spin injection. As the total effective resistance $\left(x_{0} / \sigma_{s}+\tilde{L}^{(s)} / \tilde{\sigma}_{s}\right)$ of semiconductors decreases, the spin injection efficiency increases. With increase of the current or the field, spin injection can be enhanced considerably. Moreover, in the strongfield limit, the spin injection efficiency will be determined by the total current flowing into the semiconductors.

In fact, at the zero-field limit, where $L_{u}=L_{d}=L^{(s)}$, $\tilde{L}_{u}=\tilde{L}_{d}=\tilde{L}^{(s)}$, and $x_{0} \ll L^{(s)}, \alpha\left(x_{0}\right)$ can be written as a simpler form,

$$
\alpha\left(x_{0}\right)=\left[\left(\frac{L^{(f)}}{\left(1-p_{f}^{2}\right) \sigma_{f}}+\frac{G_{\uparrow}+G_{\downarrow}}{4 G_{\uparrow} G_{\downarrow}}\right)\left(1+\frac{\sigma_{s}}{\tilde{\sigma}_{s}} \frac{x_{0} \tilde{L}^{(s)}}{\left(L^{(s)}\right)^{2}}\right)+\frac{x_{0}}{\sigma_{s}}+\frac{\tilde{L}^{(s)}}{\tilde{\sigma}_{s}}\right]^{-1}\left[\frac{L^{(f)} p_{f}}{\left(1-p_{f}^{2}\right) \sigma_{f}}+\frac{G_{\uparrow}-G_{\downarrow}}{4 G_{\uparrow} G_{\downarrow}}\right],
$$

which shows that the ratio between the total effective resistance of the two semiconductors, $x_{0} / \sigma_{s}+\tilde{L}^{(s)} / \tilde{\sigma}_{s}$, and that of the magnet, $L^{(f)} / \sigma_{f}$, together with the spinselective interfacial barrier between the ferromagnet and the semiconductor, determines the spin injection efficiency. On the other hand, at the strong-field limit, where $L_{u}=k_{B} T /|e E|, \tilde{L}_{u} \ll \tilde{L}_{d}$ and $L_{u} \ll x_{0} \ll L_{d}$, $\alpha\left(x_{0}\right)$ can be expressed in a even more compact form,

$$
\begin{aligned}
\alpha\left(x_{0}\right) & =\left[\frac{L^{(f)}}{\left(1-p_{f}^{2}\right) \sigma_{f}}+\frac{k_{B} T}{|e J|}+\frac{G_{\uparrow}+G_{\downarrow}}{4 G_{\uparrow} G_{\downarrow}}\right]^{-1} \\
& \times\left[\frac{L^{(f)} p_{f}}{\left(1-p_{f}^{2}\right) \sigma_{f}}+\frac{G_{\uparrow}-G_{\downarrow}}{4 G_{\uparrow} G_{\downarrow}}\right],
\end{aligned}
$$

which indicates that in $\mathrm{FM} / \mathrm{NS} / \mathrm{NS}$ structures spin injection is controlled by the total current flowing into the semiconductors and a distinction between the two semiconductors becomes unimportant.

\section{FIELD-DEPENDENT MAGNETORESISTANCE IN MS/NS/MS STRUCTURES}

In MS/NS/MS structures a strong positive magnetoresistance effect has been observed at low temperatures. 14 As the applied magnetic field is changed from 0 to $\sim 2 \mathrm{~T}$ the spin polarization in a magnetic semiconductor can change at low temperatures from 0 to $\sim 100 \%$.
Electric-field dependence of the magnetoresistance can be expected based on the different spin injection behaviors in the high-field regime and in the low-field regime discussed in Sec. IV. In the low-field regime, the densities of up-spin and down-spin electrons in a nonmagnetic semiconductor remain the same even in the presence of a fully spin-polarized current. Thus in the semiconductor only half of electrons contribute to the conductance if the current is $100 \%$ spin-polarized, and the resistance of the semiconductor should be twice of that for a unpolarized current. Hence the semiconductor resistance strongly depends on the spin polarization of the current in the low-field regime. In the high-field regime, however, the spin polarization of density $P$ is close to the spin polarization of current $\alpha$. Therefore if the current is $100 \%$ spin-polarized, the electron density would be also fully spin-polarized, and all electrons would contribute to the conductance. Hence in the high-field regime, the semiconductor resistance is only weakly dependent on the spin polarization, and the magnetoresistance vanishes.

Here we calculate how the magnetoresistance depends on the electric field. The magnetic semiconductor we consider here is degenerate and its spin transport is described by Eq. (2.16). Thus all results obtained in Sec. IV are also applicable to the MS/NS/MS structures. We also assume that the interfaces between the two materials are transparent, i.e., zero interface resistance. The magnetizations of the two magentic semiconductors are identical and parallel, $p_{L}=p_{R}=p(\mathbf{H})$, which are zero in the ab- 


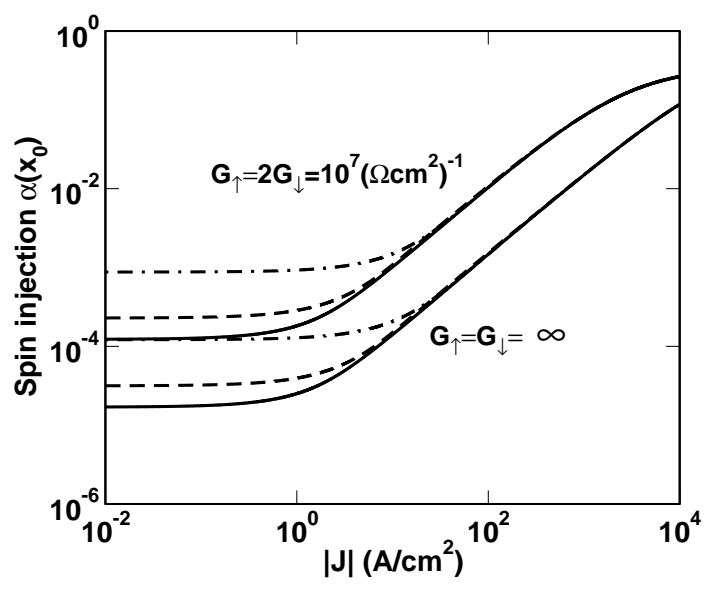

FIG. 6: Spin injection efficiency as a function of total electric current. Solid, dashed, and dot-dashed lines correspond to $\left(\sigma_{s}, \tilde{\sigma}_{s}\right)=(10,1),(1,10)$, and $(10,10)(\Omega \mathrm{cm})^{-1}$. The lower curves are for structure with transparent interface. The upper curves are for structure with a spin-selective interfacial barrier, $G_{\uparrow}=2 G_{\downarrow}=10^{7}\left(\Omega \mathrm{cm}^{2}\right)^{-1}$. Other parameters are $p_{f}=0.5, L^{(f)}=60 \mathrm{~nm}, L^{(s)}=\tilde{L}^{(s)}=2 \mu \mathrm{m}$, and $\sigma_{f}=10^{3}(\Omega$ $\mathrm{cm})^{-1}$.

sence of external magnetic field and finite for a given external magnetic field $\mathbf{H}$. The resistance of the nonmagnetic semiconductor, $R$, would depend on the spin polarization in the magnetic semiconductors because of spin accumulation at the heterostructure interfaces, and is therefore also a function of the external magnetic field.

The resistance of the nonmagnetic semiconductor can be calculated via

$$
R \equiv \frac{\mu_{0}\left(x_{0}\right)-\mu_{0}(0)}{e J}
$$

which certainly would be $R(0)=x_{0} / \sigma_{s}$ when attached to unpolarized magnetic semiconductors (zero magnetic field). The resistance in the presence of spin-polarized magnetic semiconductors with $x_{0} \ll L^{(s)}$ can be expressed as

$$
R(\mathbf{H}) \simeq x_{0} / \sigma_{s}+\frac{L^{(m)} p(\mathbf{H})}{\left[1-p^{2}(\mathbf{H})\right] \sigma_{m}}\left[2 p(\mathbf{H})-\alpha(0)-\alpha\left(x_{0}\right)\right] .
$$

Here $\alpha(0)$ and $\alpha\left(x_{0}\right)$ are the spin polarization of current at the left and right interfaces, $L^{(m)}$ the spin diffusion length in the magnetic semiconductor, and $\sigma_{m}$ the conductivity of the magnetic semiconductor. In the low-field regime, according to Eq. (4.10), we find that the magnetoresistance

$$
\frac{\Delta R}{R} \equiv \frac{R(\mathbf{H})-R(0)}{R(0)}=p^{2}(\mathbf{H})\left(1+\frac{\left[1-p^{2}(\mathbf{H})\right] \sigma_{m} x_{0}}{2 \sigma_{s} L^{(m)}}\right)^{-1} .
$$

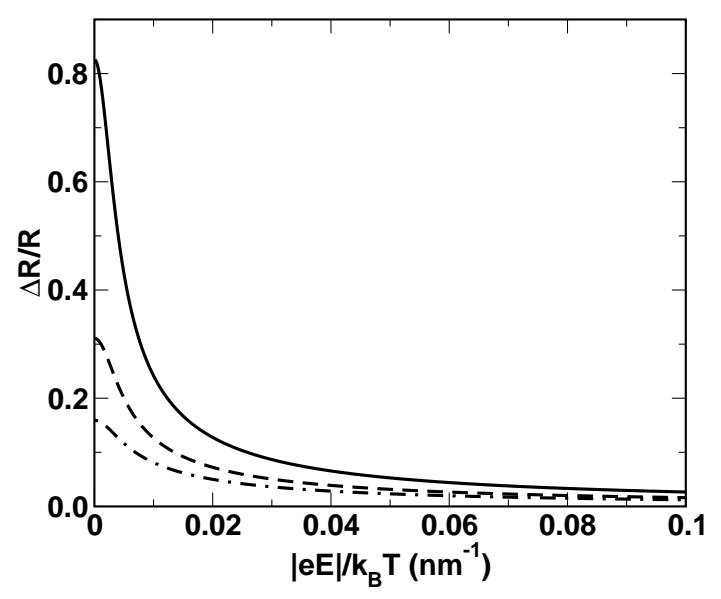

FIG. 7: Magnetoresistance $\Delta R / R$ as a function of electric field. Solid, dashed, and dot-dashed lines correspond to $p(\mathbf{H})=0.99,0.9$, and 0.8, respectively. Other parameters are $x_{0}=1 \mu \mathrm{m}, L^{(m)}=60 \mathrm{~nm}, L^{(s)}=2 \mu \mathrm{m}$, and $\sigma_{m}=\sigma_{s}$.

This can be significant for an MS/NS/MS structure because of the large $p(\mathbf{H})[p(\mathbf{H}) \sim 1]$ and small conductivity $\left(\sigma_{m} \sim \sigma_{s}\right)$ in magnetic semiconductors. We would like to point out, however, that for ferromagnetic metal/semiconductor/ferromagnetic metal structures, the magnetoresistance is usually too weak to detect due to the conductivity mismatch between metals and semiconductors $\left(\sigma_{m} \gg \sigma_{s}\right)$.

In the high-field regime, by using Eq. (4.11) we express the magnetoresistance as

$$
\frac{\Delta R}{R}=\frac{2 p^{2}(\mathbf{H})}{x_{0}}\left(\frac{1}{L_{u}}+\frac{\left[1-p^{2}(\mathbf{H})\right] \sigma_{m}}{L^{(m)} \sigma_{s}}\right)^{-1} .
$$

We see from the above expression that the effect of the electric field on the magnetoresistance can be described in terms of the field-induced up-stream spin diffusion length. Increasing the electric field will decrease the magnetoresistance because $L_{u}$ decreases with the electric field. Figure 7 illustrates the magnetoresistance as a function of electric field for an MS/NM/MS structure. We see that with increasing electric field the magnetoresistance diminishes. For example, if the parameters are chosen as follows: $x_{0}=1 \mu \mathrm{m}, L^{(m)}=60 \mathrm{~nm}$, $L^{(s)}=2 \mu \mathrm{m}, \sigma_{m}=\sigma_{s}$, and $p(\mathbf{H})=0.99$, the magnetoresistance $\Delta R / R$ decreases from $83 \%$ at zero field to $5.3 \%$ at $|e E| / k_{B} T=0.05 \mathrm{~nm}^{-1}$, or $E=125 \mathrm{~V} / \mathrm{cm}$ for $\sigma_{s}=10(\Omega \mathrm{cm})^{-1}$ at $T=3 \mathrm{~K}$.

\section{CONCLUDING REMARKS}

In summary, we have derived a general drift-diffusion equation for spin polarization in both degenerate and nondegenerate systems by consistently taking into account electric-field effects. We have demonstrated that 
as a system changes from degenerate to nondegenerate, the electric field becomes more and more important in spin transport. We have identified a high-field diffusive regime in nondegenerate semiconductors which has no analogue in metals. In this regime, there are two distinct spin diffusion lengths, i.e., up-stream and down-stream spin diffusion lengths.

We have applied this more general drift-diffusion equation for spin polarization to several typical injection structures encountered in semiconductor spintronic devices to study the spin injection behavior in these structures and the effects of electric fields. The high-field description of the spin transport in semiconductors predicts that the electric field can effectively enhance spin injection from a ferromagnet into a semiconductor. For structures with a spin-selective interfacial barrier we find that the electric field further enhances spin injection substantially. The combination of the field enhancement and the interface enhancement of spin injections may help us to obtain a comprehensive understanding of observed large spin injection in a variety of FM/NS, FM/NS/FM, and FM/NS/NS structures and current-dependent spin injection.

The consequences of spin injection into a semiconductor in the high-field regime are qualitatively different from those in the low-field regime. A high-field injection creates a notable density difference between up-spin and down-spin electrons and the spin polarization of density has a similar value as the spin polarization of current. In contrast a low-field injection does not create an appreciable density difference between up-spin and down-spin electrons, and the spin polarization of current is quite different from the spin polarization of density. One consequence of these different spin injection behaviors is that for sandwiched FM/NS/FM structures the high field destroys the symmetry between the two magnets at low fields, where both magnets are equally important to determine spin injection. The efficiency of spin injection into semiconductors in the high-field regime is "locally" determined by the magnet from which carriers are injected into the semiconductor and the magnet that collects the carriers becomes irrelevant. Another consequence is that for $\mathrm{FM} / \mathrm{NS} / \mathrm{NS}$ structures spin injection efficiency in the high-field regime is only determined by the total injected electric current and the distinction between the semiconductors becomes unimportant.

We have also examined the electric-field effect on magnetoresistance in MS/NS/MS structures. In the low-field regime, the magnetoresistance in an MS/NS/MS structure can be significant, as reported in Ref. 14. With increasing electric field, the magnetoresistance diminishes quickly. The underlying physics is that in the high-field regime the spin polarization of density is similar to the spin polarization of current in the nonmagnetic semiconductor and all electrons contribute to the conductance in the presence of spin-polarized current in contrast to half of electrons in the low-field regime if the current is fully polarized. Thus in the high-field regime, the resistance of the nonmagnetic semiconductor only weakly depends on the spin polarization of current and the magnetic field, giving rise to a diminishing magnetoresistance effect in the high-field regime.

Our calculations in this paper present a broad picture of electric field-dependent spin transport and spin injection phenomena into semiconductors. Our theory also provides physical insight into the field-induced enhancement of spin injection as well as the electric fielddependent magnetoresistance and suggests high-field injection as a simple way to amplify spin injection into semiconductor spintronic devices.

\section{Acknowledgments}

This work was supported by DARPA/ARO DAAD1901-0490.
${ }^{1}$ S. A. Wolf, D. D. Awschalom, R. A. Buhrman, J. M. Daughton, S. von Molnár, M. L. Roukes, A. Y. Chtchelkanova, and D. M. Treger, Science, 294, 1488 (2001), and references therein.

2 Semiconductor Spintronics and Quantum Computation, edited by D. D. Awschalom, N. Samarth, and D. Loss (Springer Verlag, Berlin, 2002)

3 G. Schmidt, D. Ferrand, L. W. Molenkamp, A. T. Filip, and B. J. van Wees, Phys. Rev. B 62, R4790 (2000).

4 E. I. Rashba, Phys. Rev. B 62, R16267 (2000).

5 D. L. Smith and R. N. Silver, Phys. Rev. B 64, 045323 (2001).

6 A. Fert and H. Jaffrès, Phys. Rev. B 64, 184420 (2002).

7 P. C. van Son, H. van Kempen, and P. Wyder, Phys. Rev. Lett. 58, 2271 (1987).

8 J. M. Kikkawa and D. D. Awschalom, Nature (London) 397, 139 (1999).
${ }^{9}$ I. Malajovich, J. J. Berry, N. Samarth, and D. D. Awschalom, Nature (London) 411, 770 (2001).

10 Z. G. Yu and M. E. Flatté, cond-mat/0201425.

11 M. E. Flatté and J. M. Byers, Phys. Rev. Lett. 84, 4220 (2000).

12 M. E. Flatté and G. Vignale, Appl. Phys. Lett. 78, 1273 (2001).

13 See, e.g., R. A. Smith, Semiconductors (Cambridge University Press, New York, 1978).

14 G. Schmidt, G. Richter, P. Grabs, C. Gould, D. Ferrand, and L. W. Molenkamp, Phys. Rev. Lett. 87, 227203 (2001).

15 D. T. Pierce, R. J. Celotta, G.-C. Wang, W. N. Unertl, A. Galejs, C. E. Kuyatt, and S. R. Mielczarek, Rev. Sci. Instrum. 51, 478 (1980).

16 T. Sogawa, H. Ando, and S. Ando, Phys. Rev. B 61, 5535 (2000).

17 I. Żutić, J. Fabian, S. Das Sarma, Phys. Rev. B 64, 121201 
(2001); Phys. Rev. Lett. 88, 066603 (2002).

18 J. M. Kikkawa and D. D. Awschalom, Phys. Rev. Lett. 80, 4313 (1998); J. M. Kikkawa, I. P. Smorchkova, N. Samarth, and D. D. Awschalom, Science 277, 1284 (1997).

19 S. Hershfield and H. L. Zhao, Phys. Rev. B 56, 3296 (1997).

${ }^{20}$ I. D'Amico and G. Vignale, Europhys. Lett. 55, 566 (2001).

21 A. G. Aronov and G. E. Pikus, Fiz. Tekh. Poluprovodn. 10, 1177 (1976) [Sov. Phys. Semicond. 10, 698 (1976)].

22 A. Barthélémy, A. Fert, and F. Petroff, in Handbook of Magnetic Materials, edited by K. H. J. Buschow (Elsevier Science, Amsterdam, 1999), Vol. 12.

${ }^{23}$ R. Flederling, M. Keim, G. Reuscher, W. Ossau, G. Schmidt, A. Waag, and L. W. Molenkamp, Nature (London) 402, 787 (1999).
24 B. T. Jonker, Y. D. Park, B. R. Bennett, H. D. Cheong, G. Kioseoglou, and A. Petrou, Phys. Rev. B 62, 8180 (2000).

25 Y. Ohno, D. K. Young, B. Beschoten, F. Matsukura, H. Ohno, and D. D. Awschalom, Nature (London) 402, 790 (1999).

26 H. J. Zhu, M. Ramsteiner, H. Kostial, M. Wassermeier, H. -P. Schönherr, and K. H. Ploog, Phys. Rev. Lett. 87, 016601 (2001).

27 A. T. Hanbicki, B. T. Jonker, G. Itskos, G. Kioseoglou, and A. Petrou, Appl. Phys. Lett. 60, 1240 (2002).

28 V. F. Motsnyi, V. I. Safarov, J. De Boeck, J. Das, W. Van Roy, E. Goovaerts, and G. Borghs, cond-mat/011024d. 